\title{
Performance of Differential Chaos-Shift-Keying Digital Communication Systems Over a Multipath Fading Channel With Delay Spread
}

\author{
Yongxiang Xia, Member, IEEE, Chi K. Tse, Senior Member, IEEE, and Francis C. M. Lau, Senior Member, IEEE
}

\begin{abstract}
The performance of the noncoherent differential chaos-shift-keying (DCSK) communication system over a multipath fading channel with delay spread is evaluated. Analytical expressions of the bit error rates are derived under the assumption of an independent Rayleigh fading two-ray channel model. Analytical and simulated results are presented and compared. The multipath performance of the DCSK system is also compared with that of the coherent CSK system as well as conventional generic waveform communication schemes.
\end{abstract}

Index Terms-Chaos communications, delay spread, differential chaos shift keying (DCSK), multipath Rayleigh fading channel.

\section{INTRODUCTION}

B EING wideband, chaotic signals are well suited for spread-spectrum communications [1]-[7]. Among the digital schemes proposed, chaos shift keying (CSK) and differential CSK (DCSK) have been most widely studied [8]-[11]. The former was originally proposed for a coherent implementation [3], where synchronized replicas of the chaotic basis functions are required at the receiver. However, this requirement has not yet been proven practical. The DCSK scheme, on the other hand, represents a more robust noncoherent scheme [4], in which the exact knowledge of the chaotic basis functions is not needed in the receiver. Instead, the two signal samples are correlated and the correlation result is compared with a threshold. Moroever, chaos can also be applied at the coding level, as in the chaos-based direct-sequence code-division-multiple-access (DS-CDMA) schemes studied by Mazzini et al. [5], Rovatti et al. [6], and Chen et al. [7]. In this case, since coherent communication is still applicable in the conventional sense at the waveform communication level [12], performance comparable to conventional systems can be achieved, and if further optimization at the coding level is possible with chaos, as demonstrated by Mazzini et al. [5], Rovatti et al. [6], improvement over existing DS-CDMA systems is also possible. In short, at the waveform level, all coherent schemes are impractical but their performances have benchmarking values; noncoherent schemes are more practical but their performances are inferior to conventional coherent systems.

Manuscript received March 19, 2004; revised July 14, 2004. This work was supported by the Hong Kong Research Grants Council under Grant PolyU5137/02E. This paper was recommended by Associate Editor G. Setti.

The authors are with the Department of Electronic and Information Engineering, Hong Kong Polytechnic University, Hong Kong, China (e-mail: enyxxia@eie.polyu.edu.hk; encktse@polyu.edu.hk; encmlau@polyu.edu.hk).

Digital Object Identifier 10.1109/TCSII.2004.838329
The performance of chaos-based digital communication systems under an additive white Gaussian noise (AWGN) environment has been thoroughly studied [9]-[11]. In wireless communications, however, the reflecting objects and scatterers in a wireless channel dissipate the signal energy, leading to multiple versions of the transmitted signal arriving at the receiver with different amplitudes, phases, and time delays [13], [14]. These multipath waves combine at the receiver, causing the received signal to vary greatly in amplitude and phase. Such multipath fading limits the performance in wireless applications. It is generally known that spread-spectrum systems perform significantly better than narrowband systems in a multipath environment. Since chaos-based systems are spread-spectrum systems, their performance in multipath environments should be an important practical consideration.

The multipath performance analysis and data for chaos-based communication systems at the waveform level are generally unavailable. The earliest study of multipath performance of chaos-based communication systems was performed by Kolumbán and Kis [15], [16] for the frequency-modulated DCSK (FM-DCSK) system. Their study was simulation based and each path in the two-ray channel model was assumed an ideal constant gain value. In practice, however, each path suffers from random fading, which should be duly incorporated in the channel model [14]. Recently, Mandal and Banerjee [17] analyzed the performance of the DCSK system over a channel with Rayleigh fading or Ricean fading. However, the multipath time delay has not been considered. In a spread-spectrum communication system such as DCSK, it is necessary to model the effects of multipath delay spread as well as fading. Furthermore, Mazzini et al. [18] reported a thorough study of the multipath performance of the chaos-based DS-CDMA scheme. As their system uses chaos at the code-spreading level, their results are not generally applicable to waveform communication systems using chaotic signals [12].

Our purpose here is to evaluate the performance of the DCSK system in a multipath fading channel, incorporating multipath fading for each path and the effects of delay spread. The results will be compared with the benchmark data obtained earlier for the coherent CSK system [19], and those of conventional generic waveform communication schemes.

\section{SYSTEM MODEL}

We begin with a formal description of the system to be studied in this paper. Fig. 1 shows the block diagram of the DCSK communication system. The $l$ th transmitted symbol is denoted by 


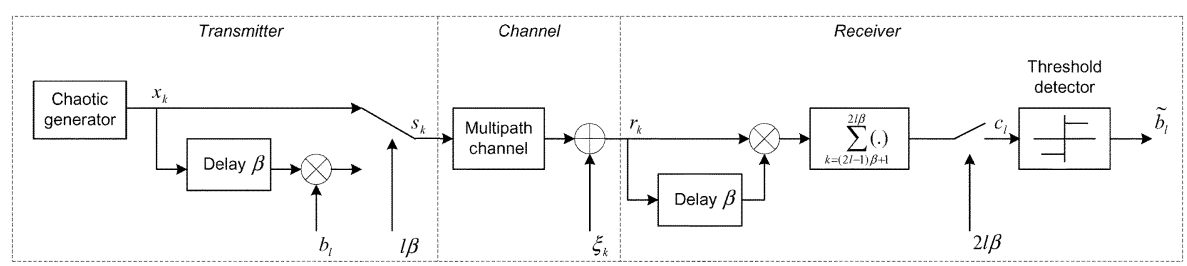

Fig. 1. Block diagram of the DCSK communication system.

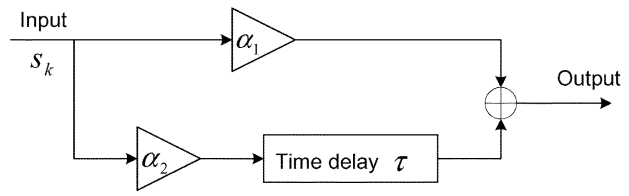

Fig. 2. Two-ray Rayleigh fading channel model.

$b_{l}$, which is either +1 or -1 , and we assume that +1 and -1 occur with equal probabilities. During the $l$ th symbol duration, the transmitted signal $s_{k}$ is

$$
s_{k}= \begin{cases}x_{k}, & k=2(l-1) \beta+1, \ldots,(2 l-1) \beta \\ b_{l} x_{k-\beta}, & k=(2 l-1) \beta+1, \ldots, 2 l \beta\end{cases}
$$

where $2 \beta$ is the spreading factor.

In spread-spectrum wireless communication systems, a commonly used channel model is the two-ray Rayleigh fading channel model [14]. Fig. 2 shows a block diagram of such a channel model. Using the discrete-time baseband equivalent model, the output of the channel is represented as

$$
\text { output }=\alpha_{1} s_{k}+\alpha_{2} s_{k-\tau}
$$

where $\alpha_{1}$ and $\alpha_{2}$ are independent and Rayleigh distributed random variables, $\tau$ is the time delay between two rays, and

$$
\begin{aligned}
& s_{k-\tau} \\
& = \begin{cases}b_{l-1} x_{k-\beta-\tau}, & k=2(l-1) \beta+1, \ldots, 2(l-1) \beta+\tau \\
x_{k-\tau}, & k=2(l-1) \beta+\tau+1, \ldots,(2 l-1) \beta \\
x_{k-\tau}, & k=(2 l-1) \beta+1, \ldots,(2 l-1) \beta+\tau \\
b_{l} x_{k-\beta-\tau}, & k=(2 l-1) \beta+\tau+1, \ldots, 2 l \beta .\end{cases}
\end{aligned}
$$

In the receiver, a correlation-based detection is used, as shown in Fig. 1. The signal received by the receiver (i.e., input to the correlator) is given by

$$
r_{k}=\alpha_{1} s_{k}+\alpha_{2} s_{k-\tau}+\xi_{k}
$$

where $\xi_{k}$ is AWGN with mean equal to zero and variance $N_{0} / 2$. Considering the $l$ th symbol, the decision variable is the output of the correlator, which is given by

$$
\begin{aligned}
& c_{l}=\sum_{k=(2 l-1) \beta+1}^{(2 l-1) \beta+\tau}\left(\alpha_{1} b_{l} x_{k-\beta}+\alpha_{2} x_{k-\tau}+\xi_{k}\right) \\
& \times\left(\alpha_{1} x_{k-\beta}+\alpha_{2} b_{l-1} x_{k-2 \beta-\tau}+\xi_{k-\beta}\right) \\
& +\sum_{k=(2 l-1) \beta+\tau+1}^{2 l \beta}\left(\alpha_{1} b_{l} x_{k-\beta}+\alpha_{2} b_{l} x_{k-\beta-\tau}+\xi_{k}\right) \\
& \times\left(\alpha_{1} x_{k-\beta}+\alpha_{2} x_{k-\beta-\tau}+\xi_{k-\beta}\right) .
\end{aligned}
$$

Then, the $l$ th decoded symbol is determined according to the following rule:

$$
\tilde{b}_{l}= \begin{cases}+1, & \text { if } c_{l} \geq 0 \\ -1, & \text { if } c_{l}<0\end{cases}
$$

In the next section, we will derive the approximated bit error rates (BERs) of the DCSK system over the two-ray Rayleigh fading channel, as defined in the foregoing.

\section{ANALYSIS OF BIT ERROR PERFORMANCE}

In the following analysis, we assume that the multipath time delay is much shorter than the bit duration, i.e., $0<\tau \ll 2 \beta$. With such an assumption, the intersymbol interference (ISI) is negligible compared with the interference within each symbol due to multipath time delay. Also, (4) may be approximated as

$$
\begin{aligned}
c_{l} \approx \sum_{k=(2 l-1) \beta+1}^{2 l \beta}\left(\alpha_{1} b_{l} x_{k-\beta}+\alpha_{2} b_{l} x_{k-\beta-\tau}+\xi_{k}\right) \\
\times\left(\alpha_{1} x_{k-\beta}+\alpha_{2} x_{k-\beta-\tau}+\xi_{k-\beta}\right) .
\end{aligned}
$$

For large $\beta$ and a given chaotic map (e.g., logistic map), we have

$$
\sum_{k=(2 l-1) \beta+1}^{2 l \beta} x_{k-\beta} x_{k-\beta-\tau} \approx 0 .
$$

Thus, $c_{l}$ may be simplified as

$$
\begin{aligned}
c_{l} \approx & \sum_{k=(2 l-1) \beta+1}^{2 l \beta} \alpha_{1}^{2} b_{l} x_{k-\beta}^{2}+\sum_{k=(2 l-1) \beta+1}^{2 l \beta} \alpha_{2}^{2} b_{l} x_{k-\beta-\tau}^{2} \\
& +\sum_{k=(2 l-1) \beta+1}^{2 l \beta}\left(\alpha_{1} x_{k-\beta}+\alpha_{2} x_{k-\beta-\tau}\right)\left(\xi_{k}+b_{l} \xi_{k-\beta}\right) \\
& +\sum_{k=(2 l-1) \beta+1}^{2 l \beta} \xi_{k} \xi_{k-\beta} \\
= & A+B+C
\end{aligned}
$$

where

$$
\begin{aligned}
& A=\sum_{k=(2 l-1) \beta+1}^{2 l \beta} \alpha_{1}^{2} b_{l} x_{k-\beta}^{2}+\sum_{k=(2 l-1) \beta+1}^{2 l \beta} \alpha_{2}^{2} b_{l} x_{k-\beta-\tau}^{2} \\
& B=\sum_{k=(2 l-1) \beta+1}^{2 l \beta}\left(\alpha_{1} x_{k-\beta}+\alpha_{2} x_{k-\beta-\tau}\right)\left(\xi_{k}+b_{l} \xi_{k-\beta}\right) \\
& C=\sum_{k=(2 l-1) \beta+1}^{2 l \beta} \xi_{k} \xi_{k-\beta} .
\end{aligned}
$$

Assuming " +1 " is transmitted (i.e., $b_{l}=+1$ ), the following statistics are easily obtained:

$$
\begin{aligned}
& E\left\{A \mid\left(\alpha_{1}, \alpha_{2}, b_{l}=+1\right)\right\}=\left(\alpha_{1}^{2}+\alpha_{2}^{2}\right) \beta E\left\{x_{k}^{2}\right\} \\
& E\left\{B \mid\left(\alpha_{1}, \alpha_{2}, b_{l}=+1\right)\right\}=E\left\{C \mid\left(\alpha_{1}, \alpha_{2}, b_{l}=+1\right)\right\}=0
\end{aligned}
$$




$$
\begin{aligned}
\operatorname{var}\left\{A \mid\left(\alpha_{1}, \alpha_{2}, b_{l}=+1\right)\right\} & =\left(\alpha_{1}^{4}+\alpha_{2}^{4}\right) \beta \operatorname{var}\left\{x_{k}^{2}\right\} \\
\operatorname{var}\left\{B \mid\left(\alpha_{1}, \alpha_{2}, b_{l}=+1\right)\right\} & =\left(\alpha_{1}^{2}+\alpha_{2}^{2}\right) \beta E\left\{x_{k}^{2}\right\} N_{0} \\
\operatorname{var}\left\{C \mid\left(\alpha_{1}, \alpha_{2}, b_{l}=+1\right)\right\} & =\frac{1}{4} \beta N_{0}^{2} \\
\operatorname{cov}\left\{A, B \mid\left(\alpha_{1}, \alpha_{2}, b_{l}=+1\right)\right\} & =\operatorname{cov}\left\{B, C \mid\left(\alpha_{1}, \alpha_{2}, b_{l}=+1\right)\right\} \\
& =\operatorname{cov}\left\{A, C \mid\left(\alpha_{1}, \alpha_{2}, b_{l}=+1\right)\right\} \\
& =0
\end{aligned}
$$

where $E[\cdot]$ and $\operatorname{var}[\cdot]$ represent the expectation and variance operators, respectively, and $\operatorname{cov}[X, Y]$ denotes the covariance of $X$ and $Y$. Then, we have

$$
\begin{aligned}
E\left\{c_{l} \mid\left(\alpha_{1}, \alpha_{2}, b_{l}=+1\right)\right\}= & \beta\left(\alpha_{1}^{2}+\alpha_{2}^{2}\right) E\left\{x_{k}^{2}\right\} \\
\operatorname{var}\left\{c_{l} \mid\left(\alpha_{1}, \alpha_{2}, b_{l}=+1\right)\right\}= & \beta\left(\alpha_{1}^{4}+\alpha_{2}^{4}\right) \operatorname{var}\left\{x_{k}^{2}\right\} \\
& +\beta\left(\alpha_{1}^{2}+\alpha_{2}^{2}\right) E\left\{x_{k}^{2}\right\} N_{0} \\
& +\frac{1}{4} \beta N_{0}^{2} .
\end{aligned}
$$

The case of sending a symbol of " -1 " may be computed in a likewise fashion, i.e.,

$$
\begin{aligned}
E\left\{c_{l} \mid\left(\alpha_{1}, \alpha_{2}, b_{l}=-1\right)\right\} & =-E\left\{c_{l} \mid\left(\alpha_{1}, \alpha_{2}, b_{l}=+1\right)\right\} \\
\operatorname{var}\left\{c_{l} \mid\left(\alpha_{1}, \alpha_{2}, b_{l}=-1\right)\right\} & =\operatorname{var}\left\{c_{l} \mid\left(\alpha_{1}, \alpha_{2}, b_{l}=+1\right)\right\} .
\end{aligned}
$$

Using (9) and (10), and assuming that $c_{l}$ follows a normal distribution under the given conditions, the conditional BER may be computed as

$$
\begin{aligned}
\operatorname{BER}\left(\alpha_{1}, \alpha_{2}\right)= & \frac{1}{2} \operatorname{Prob}\left(c_{l}<0 \mid\left(\alpha_{1}, \alpha_{2}, b_{l}=+1\right)\right) \\
& +\frac{1}{2} \operatorname{Prob}\left(c_{l} \geq 0 \mid\left(\alpha_{1}, \alpha_{2}, b_{l}=-1\right)\right) \\
= & \frac{1}{2} \operatorname{erfc}\left(\frac{E\left\{c_{l} \mid\left(\alpha_{1}, \alpha_{2}, b_{l}=+1\right)\right\}}{\sqrt{2 \operatorname{var}\left\{c_{l} \mid\left(\alpha_{1}, \alpha_{2}, b_{l}=+1\right)\right\}}}\right) \\
= & \frac{1}{2} \operatorname{erfc}\left(\left[\frac{2\left(\alpha_{1}^{4}+\alpha_{2}^{4}\right) \operatorname{var}\left\{x_{k}^{2}\right\}}{\left(\alpha_{1}^{2}+\alpha_{2}^{2}\right)^{2} \beta E^{2}\left\{x_{k}^{2}\right\}}\right.\right. \\
& \left.\left.+\frac{4 N_{0}}{\left(\alpha_{1}^{2}+\alpha_{2}^{2}\right) E_{b}}+\frac{2 \beta N_{0}^{2}}{\left(\alpha_{1}^{2}+\alpha_{2}^{2}\right)^{2} E_{b}^{2}}\right]^{-\frac{1}{2}}\right)
\end{aligned}
$$

where $E_{b}$ is the bit energy and is represented by

$$
E_{b}=2 \beta E\left\{\left(x_{k}\right)^{2}\right\}
$$

and $\operatorname{erfc}(\cdot)$, the complementary error function, is defined as

$$
\operatorname{erfc}(\psi) \equiv \frac{2}{\sqrt{\pi}} \int_{\psi}^{\infty} e^{-\lambda^{2}} d \lambda
$$

If the logistic map is used, we have

$$
\begin{aligned}
\operatorname{var}\left\{x_{k}^{2}\right\}= & \frac{1}{8} \quad \text { and } E\left\{x_{k}^{2}\right\}=\frac{1}{2} \\
\operatorname{BER}\left(\alpha_{1}, \alpha_{2}\right)= & \frac{1}{2} \operatorname{erfc}\left(\left[\frac{\left(\alpha_{1}^{4}+\alpha_{2}^{4}\right)}{\left(\alpha_{1}^{2}+\alpha_{2}^{2}\right)^{2} \beta}\right.\right. \\
& \left.\left.+\frac{4 N_{0}}{\left(\alpha_{1}^{2}+\alpha_{2}^{2}\right) E_{b}}+\frac{2 \beta N_{0}^{2}}{\left(\alpha_{1}^{2}+\alpha_{2}^{2}\right)^{2} E_{b}^{2}}\right]^{-\frac{1}{2}}\right) .
\end{aligned}
$$

For large $\beta$, the first term within the bracket in (17) may be neglected. Thus, the conditional BER may be simplified as

$$
\begin{aligned}
\operatorname{BER}\left(\alpha_{1}, \alpha_{2}\right) & =\frac{1}{2} \operatorname{erfc}\left(\left(\frac{4}{\gamma_{b}}+\frac{2 \beta}{\gamma_{b}^{2}}\right)^{-\frac{1}{2}}\right) \\
& =\frac{1}{2} \operatorname{erfc}\left(\left[\frac{4}{\gamma_{b}}\left(1+\frac{\beta}{2 \gamma_{b}}\right)\right]^{-\frac{1}{2}}\right) \\
& =\operatorname{BER}\left(\gamma_{b}\right)
\end{aligned}
$$

where $\gamma_{b}=\left(E_{b} / N_{0}\right)\left(\alpha_{1}^{2}+\alpha_{2}^{2}\right)=\gamma_{1}+\gamma_{2}, \gamma_{1}=\left(E_{b} / N_{0}\right) \alpha_{1}^{2}$, and $\gamma_{2}=\left(E_{b} / N_{0}\right) \alpha_{2}^{2}$. Denoting $\bar{\gamma}_{1}=E\left\{\gamma_{1}\right\}=$ $\left(E_{b} / N_{0}\right) E\left\{\alpha_{1}^{2}\right\}$ and $\bar{\gamma}_{2}=E\left\{\gamma_{2}\right\}=\left(E_{b} / N_{0}\right) E\left\{\alpha_{2}^{2}\right\}$, the probability density function of $\gamma_{b}$ becomes

$f\left(\gamma_{b}\right)= \begin{cases}\frac{\gamma_{b}}{\bar{\gamma}_{1}^{2}} e^{-\gamma_{b} / \bar{\gamma}_{1}}, & E\left\{\alpha_{1}^{2}\right\}=E\left\{\alpha_{2}^{2}\right\} \\ \frac{1}{\bar{\gamma}_{1}-\bar{\gamma}_{2}}\left(e^{-\gamma_{b} / \bar{\gamma}_{1}}-e^{-\gamma_{b} / \bar{\gamma}_{2}}\right), & E\left\{\alpha_{1}^{2}\right\} \neq E\left\{\alpha_{2}^{2}\right\}\end{cases}$

Finally, the BER can be obtained by averaging the conditional BER, i.e.,

$$
\operatorname{BER}=\int_{0}^{\infty} \operatorname{BER}\left(\gamma_{b}\right) f\left(\gamma_{b}\right) d \gamma_{b} .
$$

This formula will be used in the next section for evaluating the bit error performance of the system under different channel conditions.

\section{RESULTS}

We consider three cases corresponding to different path gain ratios and a fixed path delay.

Case I: The two paths have identical average power gain. In this case, the average power gain in each path is 0.5 , i.e.,

$$
E\left\{\left(\alpha_{1}\right)^{2}\right\}=E\left\{\left(\alpha_{2}\right)^{2}\right\}=\frac{1}{2} .
$$

Case II: The average power gain of the second path is $3 d B$ below that of the first path. In this case, the average powers of the two paths are

$$
E\left\{\left(\alpha_{1}\right)^{2}\right\}=\frac{2}{3} \quad \text { and } \quad E\left\{\left(\alpha_{2}\right)^{2}\right\}=\frac{1}{3} .
$$

Case III: The average power gain of the second path is $10 \mathrm{~dB}$ below that of the first path. In this case, the average powers of the two paths are

$$
E\left\{\left(\alpha_{1}\right)^{2}\right\}=\frac{10}{11} \quad \text { and } \quad E\left\{\left(\alpha_{2}\right)^{2}\right\}=\frac{1}{11} .
$$

Numerical calculation of the BERs and computer simulation of the BERs are performed. Fig. 3 shows the effect of $\beta$ for $E_{b} / N_{0}=25 \mathrm{~dB}$ and $\tau=2$. In general, BER increases with $\beta$ since for a fixed $E_{b} / N_{0}$, the noise power increases with $\beta$ and the degradation due to the increased noise power will overwhelm any gain in symbol detection that might have resulted when $\beta$ is large. In Fig. 4, the calculated and simulated BERs are plotted for $\beta=50$. We see that the approximated BERs agree well with the simulation results. The effect of $\tau$ on the BER performance is shown in Fig. 5, in which $E_{b} / N_{0}=25 \mathrm{~dB}$, 


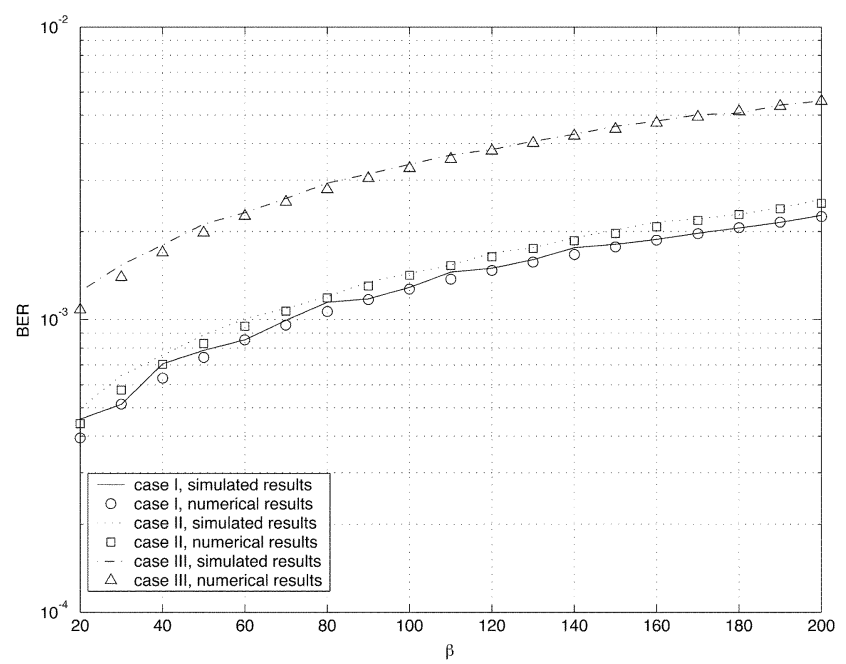

Fig. 3. BER performance of the DCSK system over a two-ray Rayleigh fading channel (BER versus $\beta$ ) with $E_{b} / N_{0}=25 \mathrm{~dB}$ and $\tau=2$.

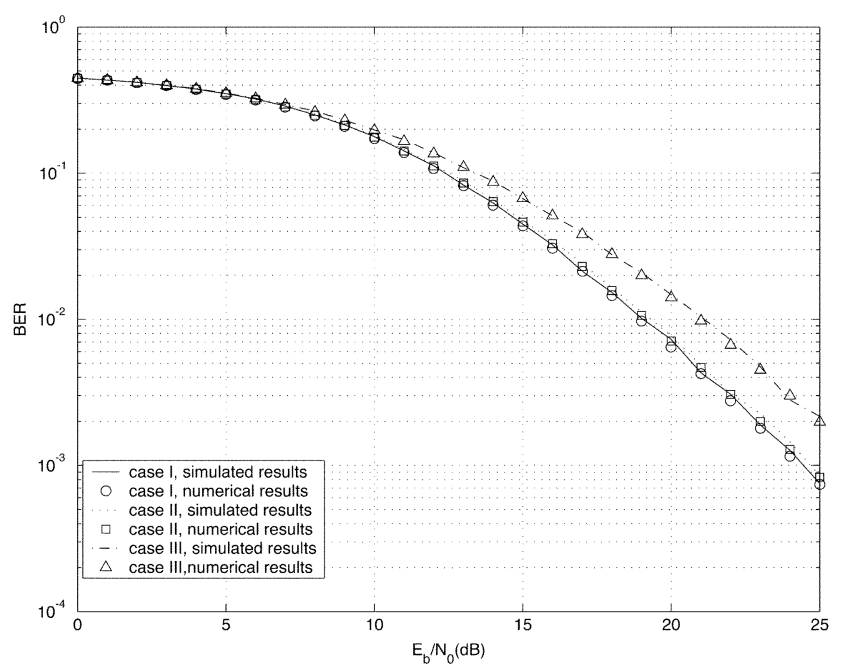

Fig. 4. BER performance of the DCSK system over a two-ray Rayleigh fading channel, with $\beta=50$ and $\tau=2$.

and $\beta=50$. Here, when $\tau$ is large, the numerical results do not agree with the simulated ones, since in the derivation of the BER, the multipath time delay is assumed to be much less than the bit duration $(\tau \ll 2 \beta)$ and hence ISI can be neglected. Under this assumption, the numerical BER result is independent of $\tau$. However, in the simulations, ISI is present and it increases with $\tau$. Thus, as $\tau$ increases, ISI increases and the simulated BER deteriorates. Fortunately, in most practical applications, the condition $\tau \ll 2 \beta$ holds, such that neglecting the ISI is justifiable [15].

\section{A. Comparison With Coherent CSK Systems}

As the DCSK system uses chaotic signals at the waveform communication level, ${ }^{1}$ performance comparison should be made appropriately with generic waveform communication systems [12]. The coherent CSK system, being a generic form of systems

${ }^{1}$ Being fundamentally different from the DCSK system, the chaos-based DS-CDMA system applies chaos at the code-spreading level [5], [6], [18], and may still employ a conventional coherent system at the waveform level. Its performance is thus not directly relevant to this study.

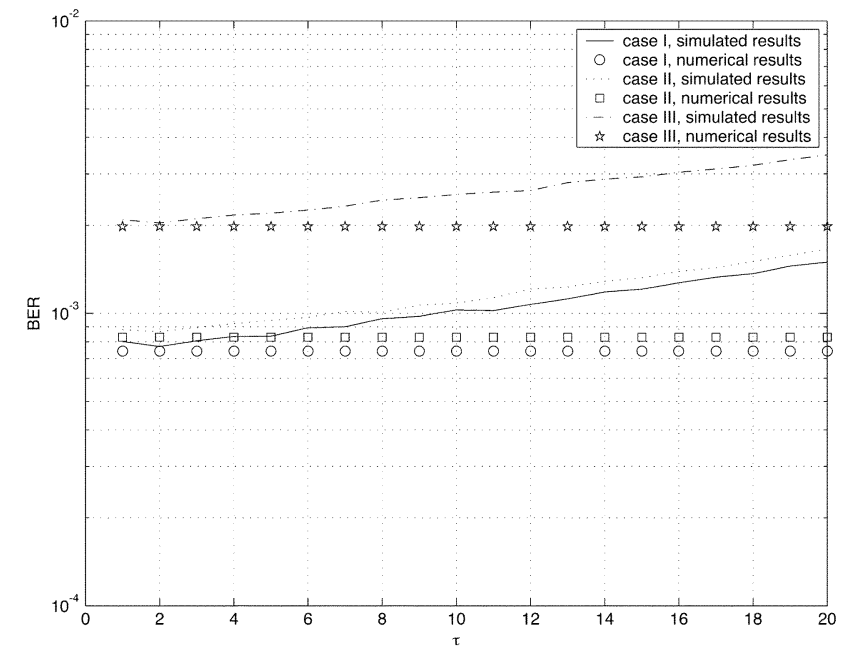

Fig. 5. BER performance of the DCSK system over a two-ray Rayleigh fading channel (BER versus $\tau$ ) with $E_{b} / N_{0}=25 \mathrm{~dB} \beta=50$.

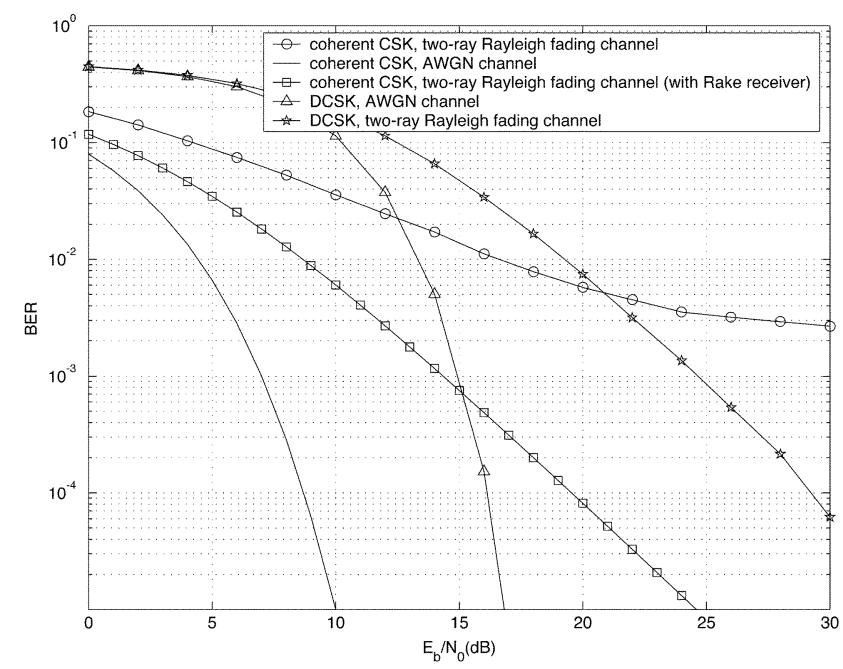

Fig. 6. Simulated results of the BER performance of the coherent CSK and noncoherent DCSK systems.

at the waveform level, may serve as a benchmark system for comparison (see Xia et al. [19]).

Fig. 6 shows the simulated results of the BER performance of the coherent CSK as well as the noncoherent DCSK over the AWGN channel and the two-ray Rayleigh fading channel. In the two-ray Rayleigh fading case, we assume that the average power gain of the second path is $3 \mathrm{~dB}$ below that of the first path. Under an AWGN channel, the coherent CSK system performs much better compared with DCSK. For example, at a BER of $10^{-3}$, the DCSK system is about $8 \mathrm{~dB}$ worse than the coherent CSK system. In a two-ray fading environment, the performance of the coherent CSK system degrades dramatically [19], but for the noncoherent DCSK, the performance degradation is less severe. Thus, the advantage of the coherent CSK system over the DCSK system diminishes. At a BER of $10^{-3}$, the DCSK system actually outperforms the coherent CSK system. The same observation is made when $E_{b} / N_{0}$ is large (say $>21 \mathrm{~dB}$ ). However, when a Rake receiver is employed in the coherent CSK system under a two-ray fading channel, the BER performance is significantly improved because the Rake receiver can combine the 


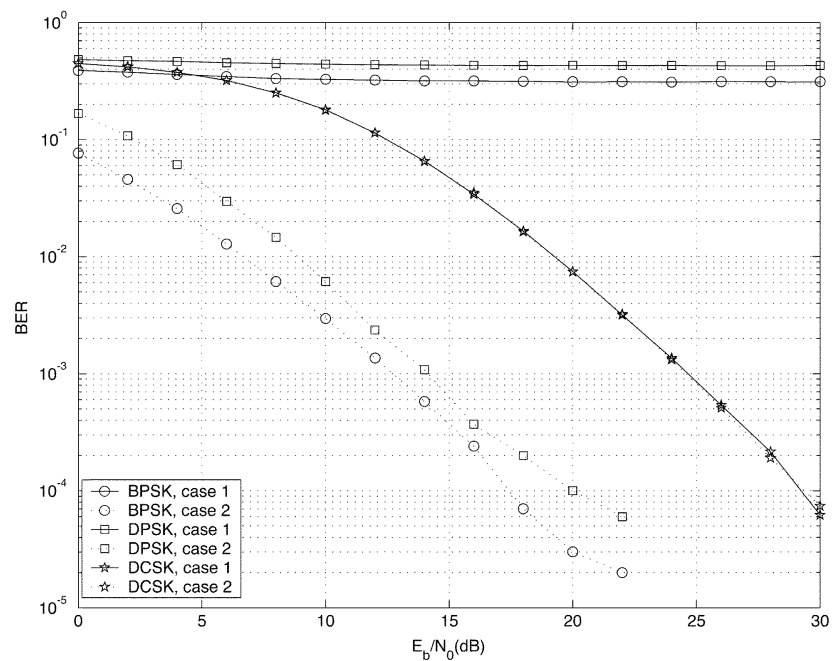

Fig. 7. Simulated results of the BER performance of coherent BPSK, noncoherent DPSK and noncoherent DCSK systems over a two-ray Rayleigh fading channel. Case 1: $\Delta \tau \omega_{c}=(2 n+1) \pi ;$ case $2: \Delta \tau \omega_{c}=2 n \pi$.

energies from the incoming rays, enhancing the signal-to-noise ratio of the signal before detection.

\section{B. Comparison With Conventional Communication Systems}

For further benchmark comparisons, we consider the conventional coherent binary phase-shift-keying (BPSK) and differential PSK (DPSK) systems. Both systems are generic waveform communication systems. In particular, the DPSK system employs a noncoherent differential detection scheme similar to the DCSK system.

The multipath performance of these systems depends on the relationship between the time delay $\Delta \tau$ and the carrier frequency $\omega_{c}$ [16]. Clearly, the worst case occurs when the signals from the two paths are out of phase, i.e.,

$$
\Delta \tau \omega_{c}=(2 n+1) \pi, \quad n=0,1,2,3, \ldots \quad(\text { case } 1)
$$

On the contrary, the best case occurs when the signals from the two paths are in phase, i.e.,

$$
\Delta \tau \omega_{c}=2 n \pi, \quad n=1,2,3, \ldots \quad(\text { case } 2)
$$

The results of these two cases are shown and compared with the DCSK case in Fig. 7. Here, we assume that the average power gain of the second path is $3 \mathrm{~dB}$ below that of the first path. The figure shows that the conventional narrowband communication systems are not suitable for the multipath fading environment since the in-phase condition is normally not met. Moreover, the multipath performances of the noncoherent DCSK system for both cases (and actually for the case of any arbitrary phase angle difference of the signals from the two paths) are identical.

\section{CONCLUSION}

The multipath performance of the noncoherent DCSK system based on a two-ray Rayleigh fading model is studied. The CSK system and two conventional communication systems are compared, as they are generic waveform communication systems and hence can provide relative indicators of how well or badly the DCSK system performs in a multipath environment with respect to the theoretical chaos-based coherent case and the conventional coherent and noncoherent cases.

\section{REFERENCES}

[1] A. Abel and W. Schwarz, "Chaos communications-Principles, schemes, and system analysis," Proc. IEEE, vol. 90, pp. 691-710, May 2002.

[2] G. Kolumbán, M. P. Kennedy, Z. Jako, and G. Kis, "Chaotic communications with correlator receivers: Theory and performance limits," Proc. IEEE, vol. 90, pp. 711-732, May 2002.

[3] H. Dedieu, M. P. Kennedy, and M. Hasler, "Chaos shift keying: Modulation and demodulation of a chaotic carrier using self-synchronizing Chua's circuits," IEEE Trans. Circuits Syst. II, vol. 40, pp. 634-642, Oct. 1993.

[4] G. Kolumbán, B. Vizvári, W. Schwarz, and A. Abel, "Differential chaos shift keying: A robust coding for chaos communication," Proc. Int. Workshop Nonlinear Dynamics of Electronic Systems, pp. 87-92, June 1996.

[5] G. Mazzini, G. Setti, and R. Rovatti, "Chaotic complex spreading sequences for asynchronous DS-CDMA-I: System modeling and results," IEEE Trans, Circuits Syst. I, vol. 44, pp. 937-947, Oct. 1997.

[6] R. Rovatti, G. Setti, and G. Mazzini, "Chaotic complex spreading sequences for asynchronous DS-CDMA-II: Some theoretical performance bounds," IEEE Trans, Circuits Syst. I, vol. 45, pp. 496-506, Apr. 1998.

[7] C.-C. Chen, K. Yao, K. Umeno, and E. Biglieri, "Design of spread-spectrum sequences using chaotic dynamical systems and ergodic theory," IEEE Trans. Circuits Syst. I, vol. 48, pp. 1110-1114, Sept. 2001.

[8] M. P. Kennedy and G. Kolumbán, "Digital communication using chaos," in Controlling Chaos and Bifurcation in Engineering Systems, G. Chen, Ed. Boca Raton, FL: CRC, 2000, pp. 477-500.

[9] F. C. M. Lau and C. K. Tse, Chaos-Based Digital Communication Systems. Heidelberg, Germany: Springer-Verlag, 2003.

[10] G. Kolumbán, "Theoretical noise performance of correlator-based chaotic communications schemes," IEEE Trans. Circuits Syst. I, vol. 47, pp. 1692-1701, Dec. 2000.

[11] - "Basis function description of chaotic modulation scheme," in Proc. Int. Workshop on Nonlinear Dynamics of Electronic Systems, Catania, Italy, May 2000, pp. 165-169.

[12] G. Kolumbán, F. C. M. Lau, and M. Small, "A new description of chaotic waveform communications: The Fourier analyzer approach," in Proc. Eur. Conf. Circuit Theory and Design, Crakow, Poland, Sept. 2003, pp. 241-244.

[13] J. G. Proakis, Digital Communications. Boston, MA: McGraw-Hill, 2001.

[14] T. S. Rappaport, Wireless Communications: Principles and Practice. Englewood Cliffs, NJ: Prentice-Hall, 1996.

[15] G. Kolumbán and G. Kis, "Multipath performance of FM-DCSK chaotic communications system," in Proc. IEEE Int. Symp. Circuits and Systems, Geneva, Switzerland, May 2000, pp. 433-436.

[16] M. P. Kennedy, G. Kolumbán, G. Kis, and Z. Jákó, "Performance evaluation of FM-DCSK modulation in multipath environments," IEEE Trans. Circuits Syst. I, vol. 47, pp. 1702-1711, Dec. 2000.

[17] S. Mandal and S. Banerjee, "Performance of differential chaos shift keying over multipath fading channels," in Proc. Indian Nat. Conf. Nonlinear Systems and Dynamics, Kharagpur, India, Dec. 2003.

[18] G. Mazzini, R. Rovatti, and G. Setti, "Chaos-based asynchronous DS-CDMA systems and enhanced rake receivers: Measuring the improvements," IEEE Trans. Circuits Syst. I, vol. 48, pp. 1445-1453, Dec. 2001.

[19] Y. Xia, C. K. Tse, and F. C. M. Lau, "Some benchmark multipath performance data of coherent CSK digital communication systems," in Proc. Int. Workshop on Nonlinear Circuits and Signal Processing, Maui, HI, Mar. 2004, pp. 261-264. 